\title{
Thermoelectric Energy Harvesting for Oxygen Determination in Refrigerated Intelligent Packaging
}

\author{
Pablo Escobedo Araque, Isabel M. Pérez de Vargas-Sansalvador, Nuria López-Ruiz, Luis Fermín Capitán-Vallvey, \\ Alberto J. Palma, Miguel A. Carvajal Rodríguez, and Antonio Martínez-Olmos ${ }^{\circledR}$
}

\begin{abstract}
In this paper, we present a passive tag for the determination of gaseous oxygen in intelligent packaging (IP). The power supply for this tag is obtained from thermoelectric energy harvesting taking advantage of the temperature difference between a cooled package and the human body. For this purpose, a compact Peltier module is attached to the surface of the package. This device is able to generate $1.2 \mathrm{~mW}$ when a temperature difference of $25{ }^{\circ} \mathrm{C}$ is applied between its surfaces. A dc-to-dc boost converter is included to generate an output voltage of $3.3 \mathrm{~V}$ and an output current of $225 \mu \mathrm{A}$ from the harvested energy by the Peltier cell, which are used to supply the measurement circuitry. A luminescent membrane sensitive to oxygen is used as a gas detector in the package. The generated signal is compared to a reference value to evaluate if the oxygen concentration inside the package falls below or above a predetermined value. This is shown by turning on a green or a red LED, respectively. The proposed system presents a resolution of $0.02 \%$ of the predicted oxygen concentration in the range of interest $(0 \%$ $5 \%$ ) and a limit of detection (LOD) of $0.007 \%$, which makes the instrument appropriate to be used in IP and active packaging (AP) technology.
\end{abstract}

Index Terms-Intelligent packaging (IP), optical oxygen sensor, passive tag, thermal energy harvesting.

\section{INTRODUCTION}

$\mathbf{O}$ VER the last decades, the technology in food packaging has been noticeably improved due to the growing interest from the consumers in properties of the product such as freshness, quality, and safety. Different approaches have been followed in order to ensure the maintenance of the properties

Manuscript received July 23,2018; revised April 1, 2019; accepted April 3, 2019. This work was supported in part by the Spanish Ministry of Economics and Competivity under Project CTQ2016-78754-C2-1-R and in part by the Unidad de Excelencia de Química aplicada a biomedicina y medioambiente, University of Granada. The work of P. E. Araque was supported by the Spanish Ministry of Education, Culture and Sport (MECD) under Grant FPU13/05032. The work of I. M. P. de Vargas-Sansalvador was supported by the European Union's Horizon 2020 research and innovation program under Grant 706303 (Multisens). The Associate Editor coordinating the review process was Dr. Branislav Djokic. (Corresponding author: Antonio MartínezOlmos.)

P. E. Araque, A. J. Palma, M. A. C. Rodríguez, and A. MartínezOlmos are with ECsens, CITIC-UGR, Department of Electronics and Computer Technology, University of Granada, 18071 Granada, Spain (e-mail: amartinez@ugr.es).

I. M. P. de Vargas-Sansalvador and L. F. Capitán-Vallvey are with ECsens, Department of Analytical Chemistry, University of Granada, 18071 Granada, Spain.

N. López-Ruiz is with the Department of Electronics Technology, University Carlos III, 28911 Leganés, Spain.

Color versions of one or more of the figures in this paper are available online at http://ieeexplore.iee.org.

Digital Object Identifier 10.1109/TIM.2019.2911757 of food or beverage or simple to inform the consumer about the state of the content. Active packaging (AP) and intelligent packaging (IP) are two of the main technologies developed with these objectives [1]. According to the European Food Safety Authority (EFSA), active food contact materials absorb or release substances in order to improve the quality of packaged food or to extend its shelf life. Intelligent food contact materials monitor the condition of packaged food or the surrounding environment, for instance, by providing information on the freshness of the food [2]. Modified atmosphere packaging (MAP) is a particular technique of AP in which the composition of the atmosphere inside the package is altered with the aim of delaying the spoilage of the content because of the bacterial activity [3].

The presence of oxygen in packaging is directly related to the loss of quality and shelf-life of food, since it is the origin of the oxidation of the content or the microbial growth [4]. The bacterial activity in the food is responsible for discoloration [5], nutritional losses [6], [7], and final spoilage [8]. In consequence, great effort has been made to include materials and devices in the packaging aimed to maintain or monitor the oxygen concentration on the inside. In AP, the use of oxygen scavenger (OS) is frequently found [9], [10]. An OS is a material in which a chemical (or combination of reactive compounds) is incorporated into a package structure and may react with oxygen to effectively remove oxygen from the inner package environment. These OS are able to reduce the concentration of oxygen to levels below $0.01 \%$ [4]. In MAP technology, a process of gas flushing substitutes the open-air atmosphere inside the package with a combination of gases where the oxygen concentration is reduced to values in the range $0.5 \%-5 \%$ [11]. In an alternative approach known as high-oxygen MAP, the oxygen concentration is not reduced but increased to levels higher than $70 \%$ [12]. In IP, the concentration of oxygen is monitored and related to the state of the packaged food or beverage [13], [14].

In all of these techniques, the use of oxygen indicators and sensors becomes a necessity. Many examples have been reported for both oxygen indicators [15]-[17] and sensors [18]-[20], being very adequate those based on luminescent oxygen sensitive elements [21]-[23] because they are inexpensive, nontoxic, tuneable and they exhibit a long ambient shelf-life [13].

For reading of the oxygen indicator and transmission of the information to the user, it is usual in IP and AP technologies to include electronic circuitry attached to the package. 
These electronics must be very reduced in terms of size and power consumption. In this field, radiofrequency identification (RFID) and, more recently, near-field communication (NFC) has been adopted as major technologies in the development of tags included or directly printed on the packaging. The reading of the gas detectors and the data transmission is carried out by a remote RFID/NFC reader [24], [25]. The main advantage of these protocols lies in the fact that they are able to provide the tag with a passive character. Therefore, many examples of passive RFID-/NFC-based tags for oxygen and other gases monitoring have been proposed in IP and AP [26]-[28]. As an alternative to predominant RFID-based tags, other schemes of passive tags have been conceived to be included [29], [30].

In this paper, we present a novel platform with a passive tag with sensing capability intended to be used in IP and AP. It is based on the harvesting of thermal energy by means of a thermoelectric generator when a temperature difference is applied. This thermal gap can be found between the surface of a cooled package (stored in a refrigerator at low temperature) and the user's skin. With this idea, a Peltier module is used in the design to generate power to supply a low-consumption measurement circuit when one of its surfaces is attached to the cooled package and the other is pressed by the bare finger of a user. To the authors' knowledge, this is the first report of a passive tag designed for the determination of oxygen in IP and AP where the power supply relies only on thermoelectric energy harvesting, and no other source of power supply such as batteries or near-field energy harvesting is used. The measurement circuit is aimed to determine if the oxygen concentration inside the package remains within a predefined safety limit. The result is visually transmitted to the user by turning on a green or a red LED depending on the oxygen concentration.

\section{A. Reagents and Materials}

Platinum octaethylporphyrin (PtOEP) complex, 1, 4-diazabicyclo[2.2.2] octane (DABCO, 98\%), tetrahydrofuran (THF), and polystyrene (PS), average MW 280000; $\mathrm{Tg}, 100{ }^{\circ} \mathrm{C}$; GPC grade (Sigma-Aldrich Química S.A., Spain) were used as reagents for the preparation of the oxygen-sensitive membranes.

The tag was screen-printed using a Serfix III screen printing machine (Seglevint SL, Spain) on $250-\mu$ m-thick flexible polyethylene terephthalate substrate (PET ES301450, Goodfellow Cambridge Ltd., England), which has high optical transmission $(>85 \%)$ in the visible spectrum. The screen mesh used for printing consists of an aluminum rectangular structure with a mesh density of 150 Nylon threads per centimeter $(\mathrm{T} / \mathrm{cm})$. The circuit layout is printed using the conductive silver-based ink SunTronic CRSN 2442 (Sun Chemical, USA). After printing, a thermal sintering process was carried out at $120{ }^{\circ} \mathrm{C}$ during 5 min in a convection air oven Venticell VC55 (MMM Medcenter Einrichtungen GmbH, Germany). The chips and external components were attached to the substrate using the CircuitWorks CW2400 conductive epoxy (Chemtronics,
Kennesaw, USA). This silver epoxy allows room temperature sintering and has a volume resistivity lower to $0.001 \Omega \cdot \mathrm{cm}$ after being cured.

\section{B. Instruments and Software}

The electrical characterization and validation of the developed tag was carried out using the following laboratory instrumentation: mixed-signal oscilloscope (MSO4101, Tektronix, USA), 81-/2-bit Digital Multimeter 3158A (Agilent Technologies, USA), 15-MHz waveform generator 33120A (Agilent Technologies), DC power supply E3630A (Agilent Technologies), and a balance DV215CD (Ohaus Co., USA). The standard mixtures for instrument calibration and characterization were prepared using $\mathrm{N}_{2}$ as the inert gas by controlling the flow rates of different high purity gases $\mathrm{O}_{2}$, and $\mathrm{N}_{2}$, entering a mixing chamber (Air Liquid España S.A., Spain) operating at a total pressure of 760 torr and a flow rate of $500 \mathrm{~cm}^{3} \mathrm{~min}^{-1}$. This mixing chamber contains several computer-controlled mass flow controllers model F-201C-RAB-00-V (Bronkhorst High-Tech B.V, The Netherlands) that provide an accurate flow of the selected gases. The oxygen concentration is measured using the reference commercial instrument CheckPoint-Handheld Gas Analyzer $\left(\mathrm{O}_{2} / \mathrm{CO}_{2}\right)$ Dansensor A/S (Rønnedevej 18, DK-4100 Ringsted, Denmark).

\section{System Description}

1) Oxygen Sensitive Membrane: Sensing membranes containing luminophore for $\mathrm{O}_{2}$ were prepared from a cocktail that contains $50 \mathrm{mg}$ of PS dissolved in a $1 \mathrm{~mL}$ of freshly distilled THF, using an ultrasonic bath, 0.5-mg PtOEP, and 12-mg DABCO. The sensor preparation consists of the casting on one side of the substrate that acts as a package envelope with $20 \mu \mathrm{L}$ of the cocktail. This support might be the flexible substrate of the developed tag or the inner surface. After that, the support was left to dry in darkness in a saturated THF atmosphere for $1 \mathrm{~h}$ at room temperature. The response and recovering times of the sensitive membrane are 5 and $10 \mathrm{~s}$, respectively [31], [32].

2) Description of the Sensing Tag: As stated in Section I, the goal of this paper is to develop a flexible passive tag intended to be used in AP and IP with the objective of determining if the oxygen concentration inside the package is above or below a predetermined threshold. The challenge is to obtain the power supply for the required electronics from thermal energy harvesting as an alternative to the widely implemented RFID technology in this kind of applications. This approach presents some benefits over the RFID-/NFC-based tags, such as the elimination of the large-size antenna needed in these technologies and the use of an external reader. As it happens in other passive systems, the power availability is very limited; therefore, an ultralow power consumption design is required. In previous works by the authors where other designs of passive tags for oxygen determination were presented, the measurement electronics was purely digital [30], [34]. That approach implied the inclusion of a microcontroller unit for signal processing and the implementation of the thermal drift 


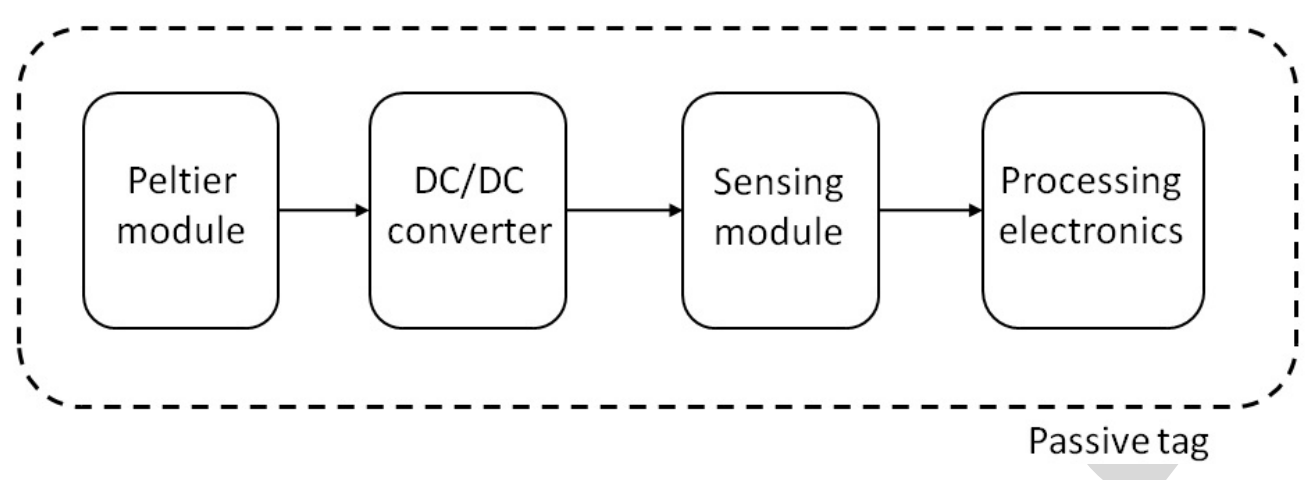

Fig. 1. Block diagram of the passive sensing tag.

correction. Although this solution provided more capabilities to the system, it makes the design more complex and also increased the power consumption. The proposed measurement system is aimed to operate in refrigerated packages stored at a stable temperature of $4{ }^{\circ} \mathrm{C}$. This means that there are no temperature changes in the package; thus, no thermal drift of the oxygen-sensitive membrane has to be corrected. In this situation, the digital design of the previous works is replaced by low-power analog electronics that allows a simpler design.

The block diagram of the developed sensing tag is shown in Fig. 1.

TES1-03102 (Merit Tech Group, China) was used as Peltier cell in this prototype, selected because of its compact dimensions of $15 \times 15 \mathrm{~mm}^{2}$ (surface) and $3.8 \mathrm{~mm}$ (thickness). It generated a low voltage and current between its terminals when a temperature difference is applied between its surfaces. This thermoelectric voltage was elevated by means of a dc-todc boost converter based on the integrated circuit LTC3108 (Linear Technology Corp., USA). This device was able to operate from voltage inputs as low as $20 \mathrm{mV}$ and provided a selectable output voltage of $2.35,3.3,4.1$, or $5 \mathrm{~V}$. The connection scheme of this device for the implementation of a dc-to-dc step-up converter is described in the datasheet of the manufacturer [35]. This stage requires a transformer with a turn ratio of $1: 100$. For this purpose, the integrated transformer model LPR6235-752SMR (Coilcraft Inc., USA) has been selected.

The sensing module is depicted in Fig. 2(a). The luminophore used as oxygen sensitive membrane has been already widely described in [33]. It is known that when this luminophore is optically excited at certain wavelengths (380 and $533 \mathrm{~nm}$ ), it emits luminescence in the red region of the spectrum at $650 \mathrm{~nm}$. In this paper, the selected excitation light source was an LED model SMP2-UPGC (Bivar Inc., California, USA) with peak emission at $536 \mathrm{~nm}$. As shown in Fig. 2(a), this LED was biased at a constant voltage of $2.5 \mathrm{~V}$ thanks to a reference diode model LM285-2.5-N (Texas Instrument Incorporated, USA). The optical response of the membrane was registered using a color detector model S6430-01 (Hamamatsu Photonics K.K., Japan). This device detects monochromatic red light $(\lambda \mathrm{p}=660 \mathrm{~nm})$ and generates an output current which varies linearly with the intensity of the incident light.

The current generated in the sensing module was then processed by the processing electronics of Fig. 2(b). First, the current was converted to voltage in a first stage formed by the operational amplifier A1 model MCP6242 (Microchip Technology Inc., USA) and the feedback resistor $R_{o}$. This device was selected due to its low bias and offset current (1 pA typical) since the current generated by the color detector was in the nanoamperes range. The output voltage of A1 was then compared by means of the operational amplifier A2 to a reference value generated in the voltage divider formed by the resistances $R_{a}$ and $R_{b}$. This reference value was adjusted to correspond to the oxygen concentration considered as the safety limit in a given application, for example, $2 \%$ in MAP or $0.01 \%$ in OS-based AP. Depending on the result of the comparison, the A2 output voltage was $V_{\mathrm{cc}}$ or $0 \mathrm{~V}$, therefore activating the green or the red LED, respectively. The status of these LEDs informs the user in a visual code about the oxygen concentration inside the package.

Fig. 3 presents a photograph of the developed tag. The presented system was fabricated on a flexible screen-printed tag of reduced dimensions $60 \times 30 \mathrm{~mm}^{2}$ (not including the Peltier cell), which can be attached to the package external surface. The oxygen sensitive membrane can be directly printed or deposited on the inner surface of the envelope since this luminophore shows very low toxicity and it is not in contact with food [36]. The excitation LED and the color detector can be placed facing the membrane, following the scheme proposed in [30] and [34].

\section{RESUlTS AND DISCUSSION}

As has been described above, the developed passive tag is intended to be power supplied from the harvesting of thermal energy. A Peltier module is included in this design as the harvester element. This device requires a temperature difference between its surfaces to generate power. In this paper, we have taken advantage of the thermal gap existing between a cooled package stored in a refrigerator at a reference temperature of $4{ }^{\circ} \mathrm{C}-5{ }^{\circ} \mathrm{C}$ and the body temperature of a user. If we assume that the skin of the finger is at $30{ }^{\circ} \mathrm{C}$, a temperature difference of at least $25^{\circ} \mathrm{C}$ can be achieved if the cold side of the Peltier module is attached to the cooled package and the hot side is pressed by a user using his bare finger.

The Peltier module included in the proposed system has been characterized by the application of different temperature gradients in order to evaluate the generated voltage. The obtained results are shown in the curve of Fig. 4. As can be 
A
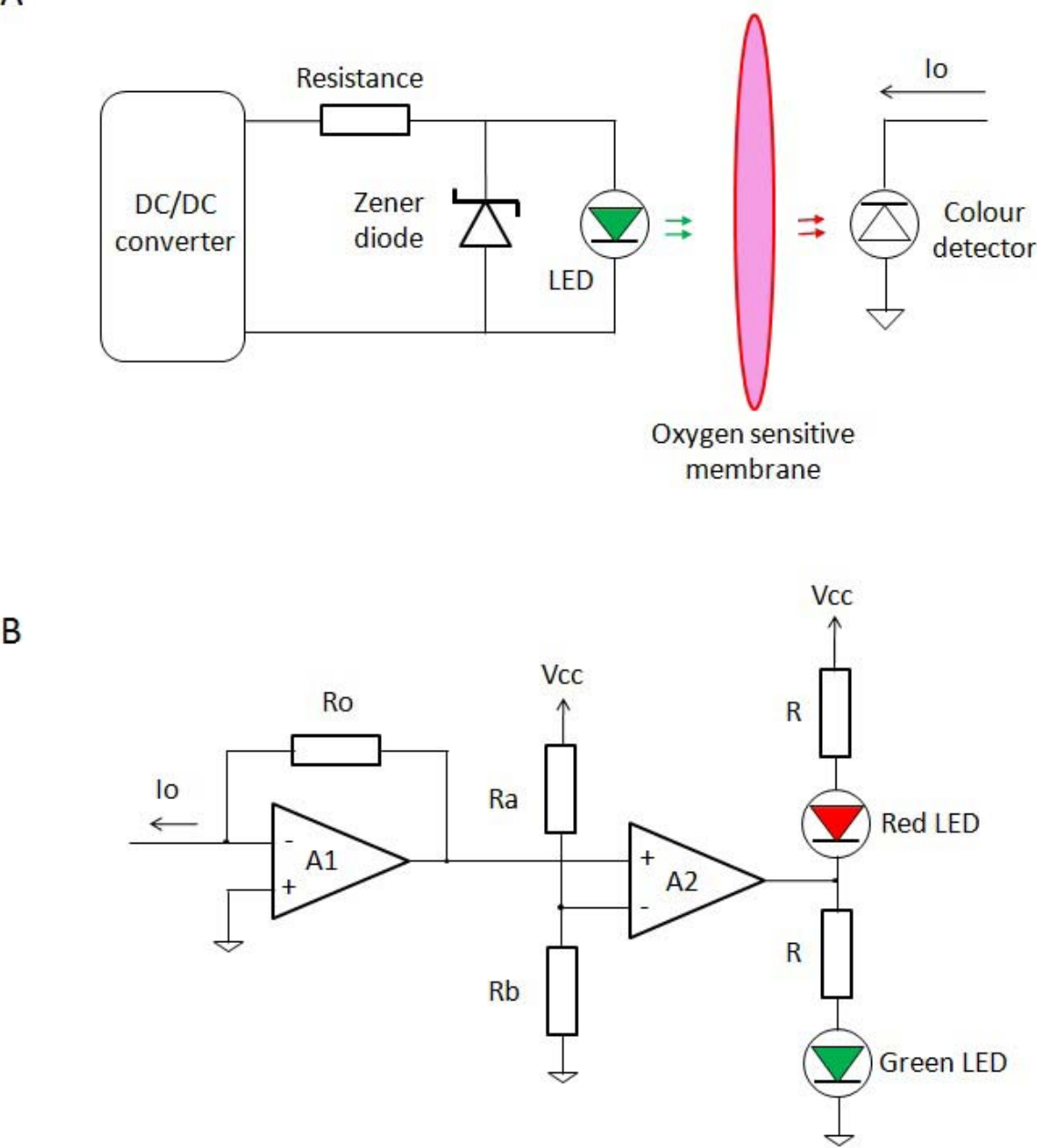

Fig. 2. Scheme of the (a) sensing module and (b) processing electronics.

seen, the voltage across the cell follows the expected linear behavior in response to increasing temperature differences. The Peltier cell is able to generate an output voltage of $120 \mathrm{mV}$ if a temperature gradient of $25^{\circ} \mathrm{C}$ is applied.

The output voltage from the Peltier cell is the input to the dc-dc converter, which generates a stable rectified voltage of $3.3 \mathrm{~V}$ even if the input voltage suffers low variations. Therefore, fluctuations of the generated voltage from the Peltier cell caused by temperature variations do not affect the regulated output voltage that provides the powering to the tag. The only scenario where the tag would not work occurs in the case that the input voltage of the dc-dc converter is not high enough to provide a stable rectified output voltage of $3.3 \mathrm{~V}$. This would happen if the temperature difference between both sides of the Peltier cell is not high enough to provide the required input voltage. We have experimentally measured the minimum temperature difference to obtain the stable rectified voltage of $3.3 \mathrm{~V}$ at the output of the dc-dc converter, obtaining a value of $8{ }^{\circ} \mathrm{C}$. In our proposed application, the temperature difference is around $25^{\circ} \mathrm{C}$, so the system will work properly and little temperature variations would not affect the performance of the system.

When it is connected to the tag, a power of $1.2 \mathrm{~mW}$ is supplied by the Peltier cell for a temperature difference of $25{ }^{\circ} \mathrm{C}$. The total current requirement for the measurement circuit depicted in Fig. 2 is $225 \mu \mathrm{A}$ when the boost converter is configured to provide an output voltage of $3.3 \mathrm{~V}$. Therefore, the power consumption of the measurement electronics in the tag is $743 \mu \mathrm{W}$. This power requirement has been reduced in comparison with previous passive tags developed by the authors, which were 1.6 and $12 \mathrm{~mW}$ [30], [34]. As it can be deduced, the Peltier module under the temperature difference available in this application generates a power high enough to supply the tag, which is designed for ultralow power consumption.

This system has been calibrated in the full range of oxygen concentrations $0 \%-100 \%$, with six replicas for each concentration at a fixed temperature of $5{ }^{\circ} \mathrm{C}$ to simulate refrigerator conditions. The experimental setup disposed for this calibration is presented in Fig. 5.

The desired oxygen concentration is generated by mixing the gases $\mathrm{N}_{2}$ and $\mathrm{O}_{2}$ in the mixing chamber with different mass flow rates. In Table I, some examples of the mass flow rate for each gas required to generate different oxygen concentrations are presented.

The results are shown in Fig. 6(a), where the output voltage of the operational amplifier A1 in Fig. 2(b) is represented versus the oxygen concentration. In Fig. 6(b), only the range 


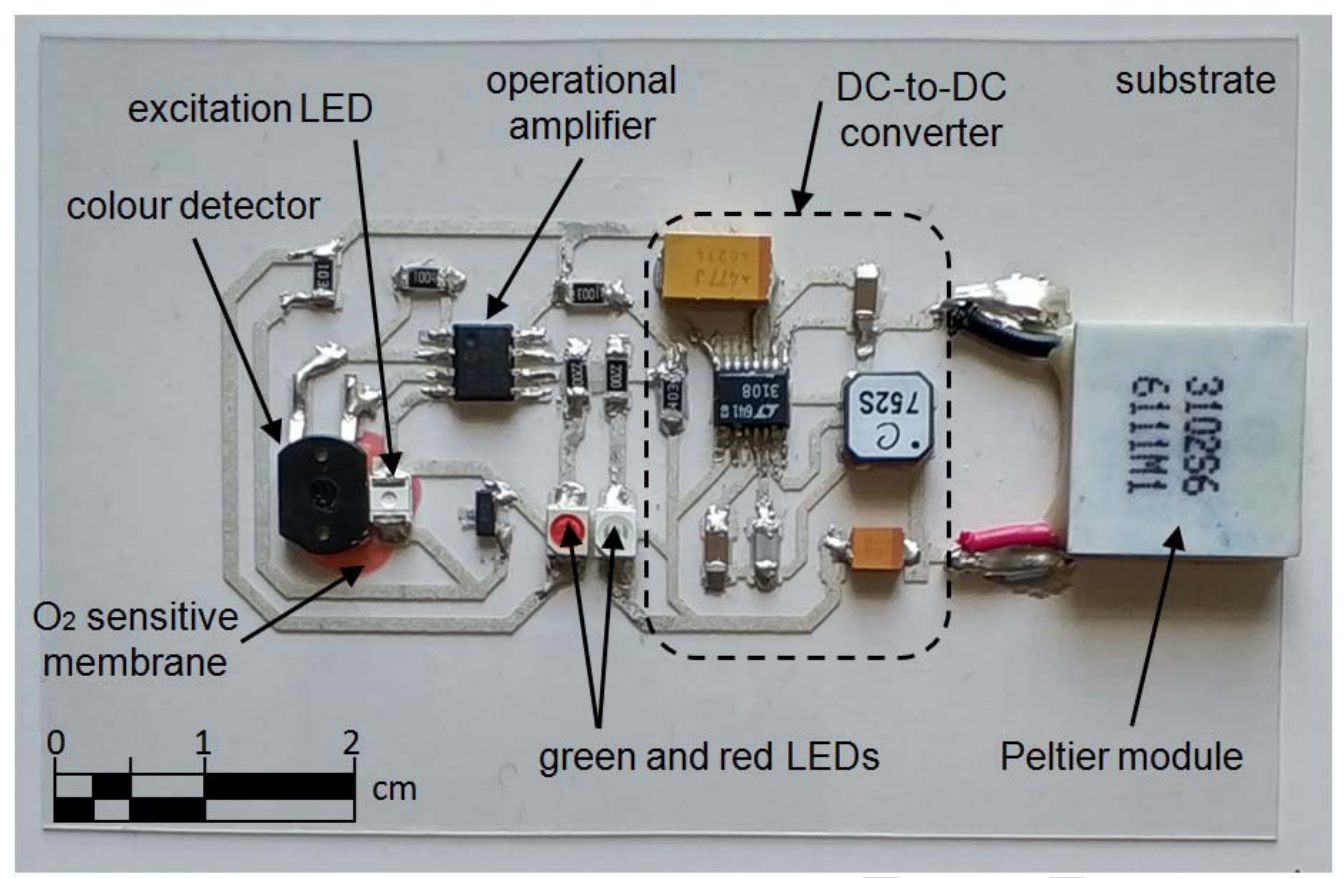

Fig. 3. Photograph of the developed tag pointing out the electronic components, Peltier cell, and the optochemical sensing membrane.

TABLE I

Mass Flow RATES FOR DifFERENT OXYGEn CONCENTRATIONS IN THE GAS Flow

\begin{tabular}{|c|c|c|c|}
\hline $\mathrm{N}_{2}(\%)$ & $\mathrm{O}_{2}(\%)$ & Mass flow rate $\mathrm{N}_{2}\left(\mathrm{~cm}^{3} / \mathrm{min}\right)$ & Mass flow rate $\mathrm{O}_{2}\left(\mathrm{~cm}^{3} / \mathrm{min}\right)$ \\
\hline 100 & 0 & 5.00 & 0.00 \\
\hline 98 & 2 & 4.90 & 0.10 \\
\hline 95 & 5 & 4.75 & 0.25 \\
\hline 90 & 10 & 4.50 & 0.50 \\
\hline 79 & 21 & 3.95 & 1.05 \\
\hline 70 & 30 & 3.50 & 1.50 \\
\hline 50 & 50 & 2.50 & 2.50 \\
\hline 25 & 75 & 1.25 & 3.75 \\
\hline 0 & 100 & 0.00 & 5.00 \\
\hline
\end{tabular}

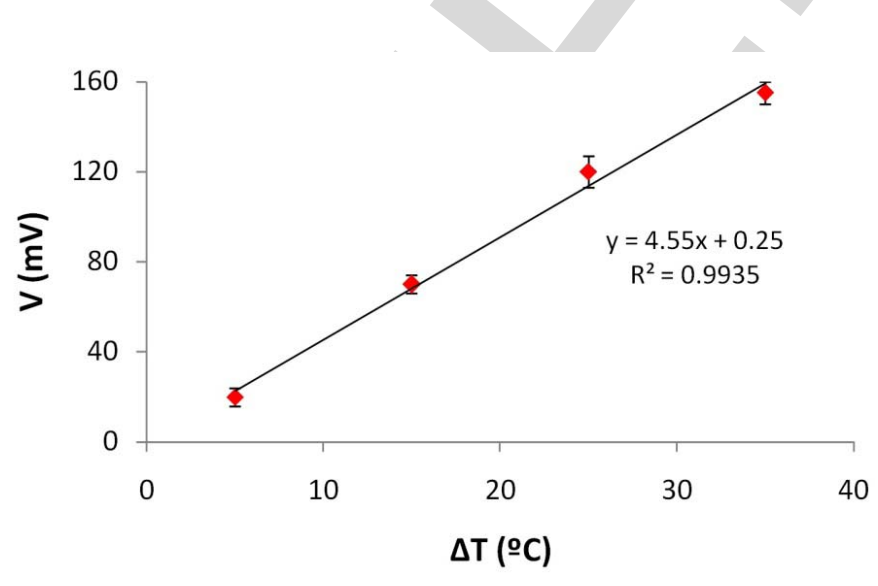

Fig. 4. Output voltage from the Peltier cell as a function of the temperature difference between both cell surfaces. of interest $0 \%-5 \%$ and the corresponding fitting curve are presented. Error bars are included in the graphics, but they are too small to be appreciated.
The obtained data were fitted to a potential curve in the form $V \mathrm{o}=\alpha \cdot\left[\mathrm{O}_{2}\right]^{\beta}$ [37], with $\alpha=577.69$ and $\beta=-0.461$ for the full range and $\alpha=350.72$ and $\beta=-0.168$ for the reduced range $0 \%-5 \%$ of oxygen, being the coefficient of correlation $R^{2}$ above 0.99 in both cases. From this calibration curve, it was possible to obtain the appropriate reference value to be used in the comparator of Fig. 2(b), since it corresponds to the oxygen concentration that is considered as a safety limit in a particular application.

The resolution of this system was obtained from the potential curves found in the fitting of the experimental data, taking derivatives in both sides of the equation and approximating these derivatives to increments [37]

$$
\Delta O_{2}=\frac{\left(\frac{V_{o}}{\alpha}\right)^{1 / \beta}}{\beta V_{o}} \Delta V_{o}
$$

where $\Delta V o$ is the error or uncertainty in the determination of the output voltage of the operational amplifier A1. This uncertainty was evaluated as the standard deviation of the replicas 
A

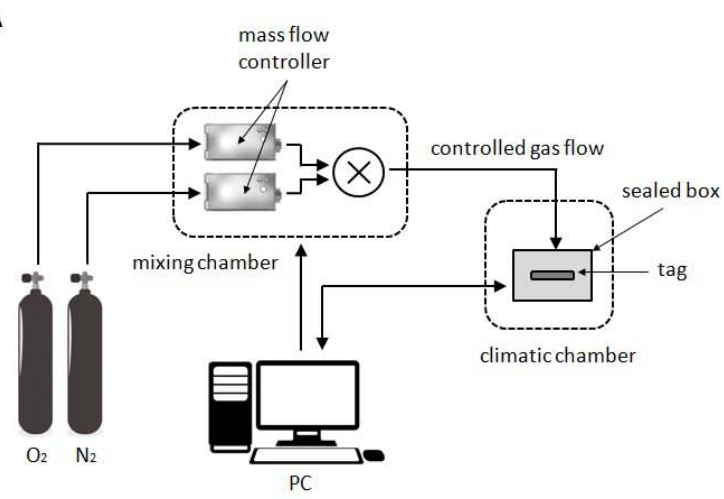

B
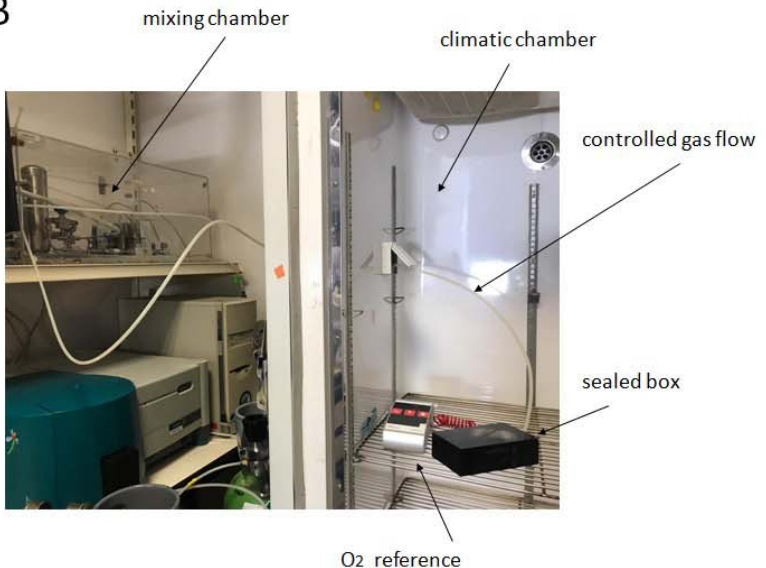

Fig. 5. (a) Scheme and (b) photography of the experimental setup.

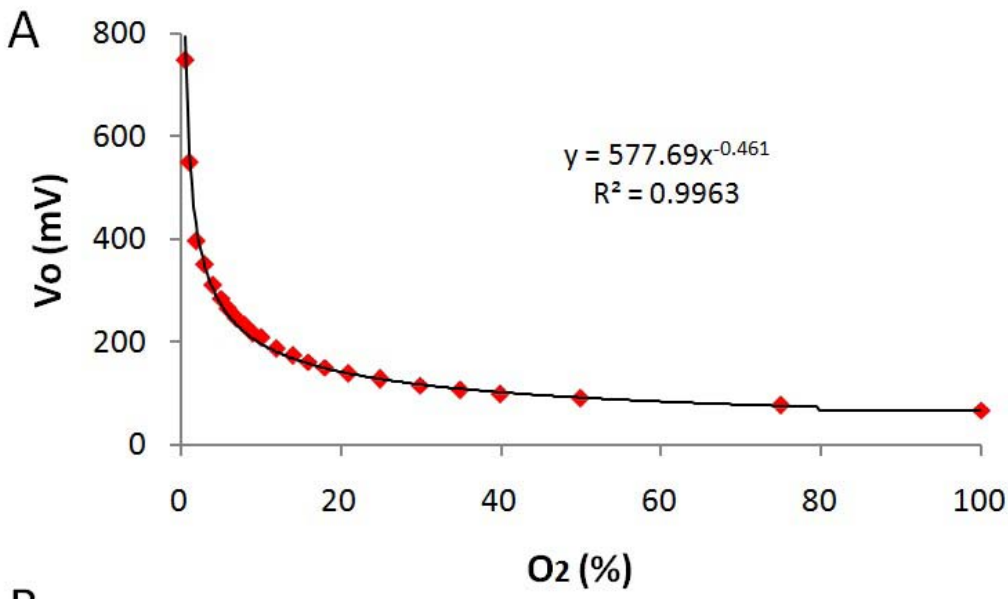

$\mathrm{B}$

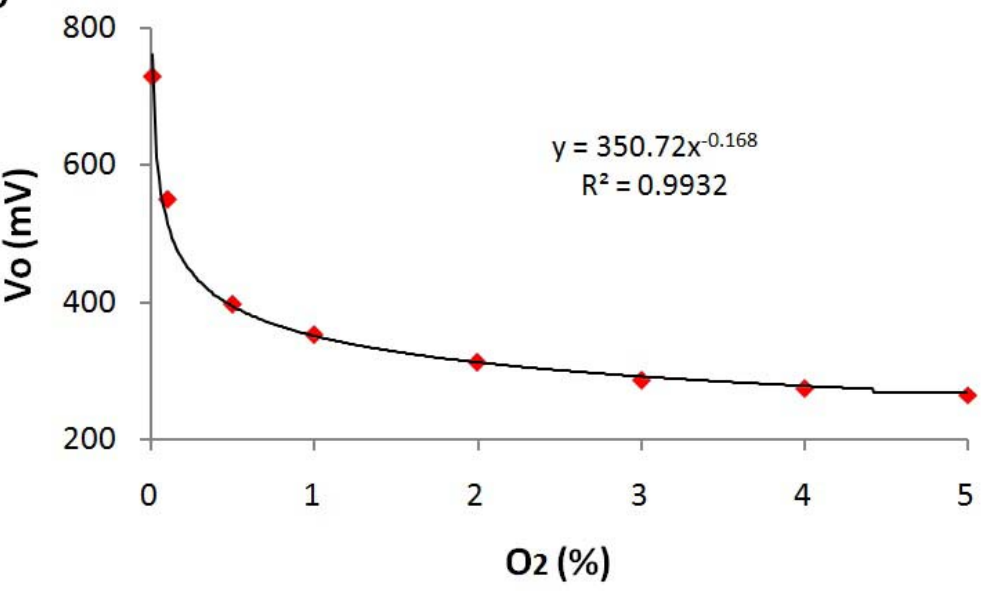

Fig. 6. Calibration curve of the measurement system in the (a) full range and (b) reduced range $0 \%-5 \%$.

taken for the measurement of each oxygen concentration in the calibration process. The resolution obtained as expressed in (1) obviously depends on the oxygen concentration; therefore, distinct values of the resolution were achieved for different oxygen concentrations [34]. The mean resolution obtained is $0.4 \%$ of oxygen concentration in the full range. This value was reduced to $0.02 \%$ if only the range $0 \%-5 \%$ of $\mathrm{O}_{2}$ is considered, which is the usual range of interest in IP and AP applications.

The limit of detection (LOD) was calculated following the standard criteria: LOD $=y_{b}+3 s_{b}$, where $y_{b}$ is the average blank signal and $s_{b}$ is the standard deviation of the blank, which was determined using a high number of replicas (above 20) [38]. The obtained value in our case was $0.007 \%$ (70 ppm). 
Agilent Technologies

WED JAN 16 19:42:19 2019

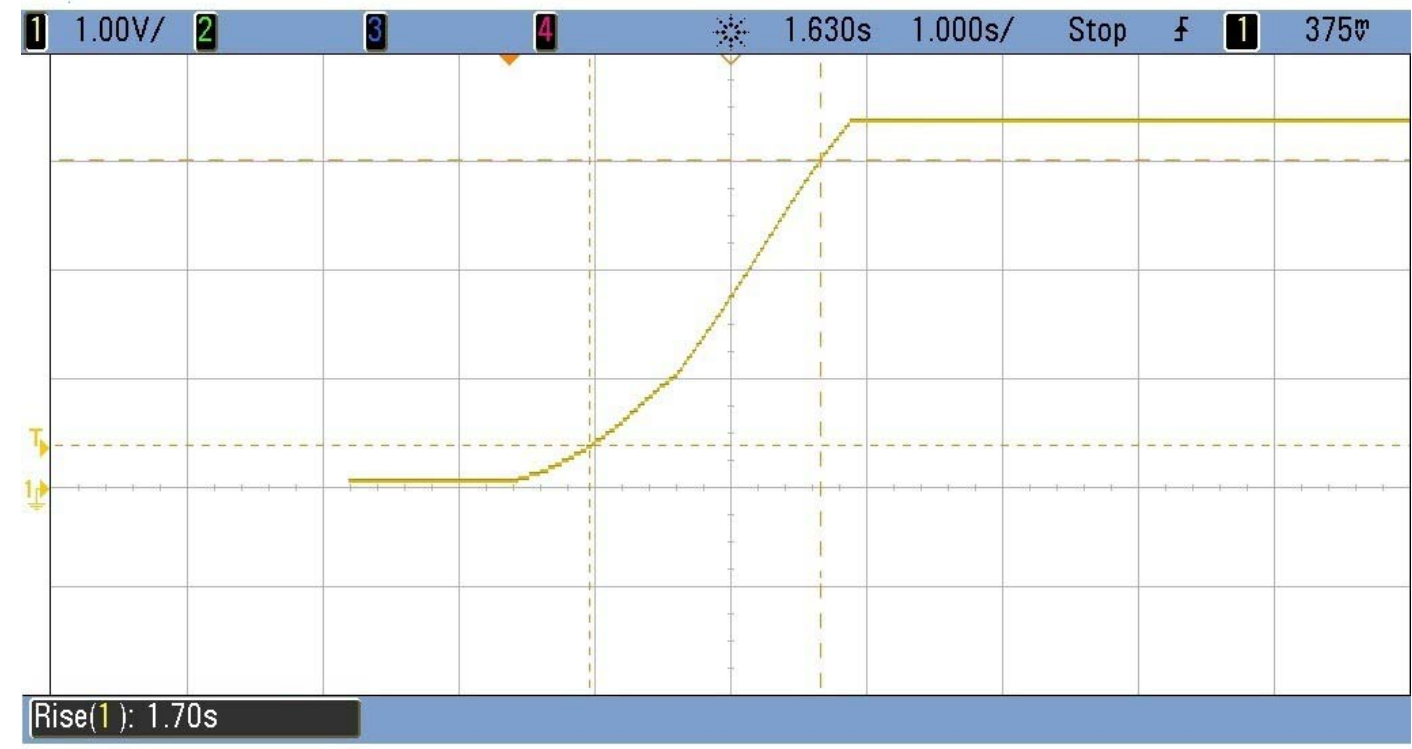

Fig. 7. Output voltage from the dc/dc converter.

As can be seen, both values of resolution and LOD are plenty compatible with applications in AP and IP, where values of oxygen concentration as low as $0.01 \%$ are required.

The response time of the electronics was limited by the design of the dc-to-dc boost converter integrated in the presented tag. Since it operated with very low voltages and currents, a time in the range of seconds was required to provide a stable output voltage of $3.3 \mathrm{~V}$. In this case, the rise time of this magnitude was $1.7 \mathrm{~s}$, as it is shown in Fig. 7, where the regulated output from the $\mathrm{dc} / \mathrm{dc}$ stage is presented. The voltage starts rising when the finger is pressed to the Peltier cell. Once the tag is powered, the response of the electronics to changes in oxygen concentration, that is, changes in the luminescence generated by the oxygen-sensitive membrane is in the order of microseconds (according to manufacturers, the rise time of the color detector S6430-01 is $0.5 \mu \mathrm{s}$ and the slew rate of the operational amplifiers MCP6242 is $0.3 \mathrm{~V} / \mu \mathrm{s}$ ). Therefore, the response and recovery time of the instrument is mainly limited by the oxygen sensitive membrane.

\section{CONCLUSION}

The presented work describes a passive tag designed for oxygen monitoring in IP applications. The main novelty of the proposed system relies on the technique applied for its powering, which is generated from thermoelectric energy harvesting. The use of thermal energy for the power supply in IP and AP is reported here for the first time. A compact Peltier module was included in the package attached to the envelope as the thermal energy harvester along with a miniaturized step-up converter circuit. When the package is stably refrigerated at $4{ }^{\circ} \mathrm{C}-5^{\circ} \mathrm{C}$, a temperature gap of $25{ }^{\circ} \mathrm{C}$ is induced between both sides of the Peltier module by simply pressing the shown side with the bare finger of a user. This temperature gradient was enough to power the measurement circuit since the minimum temperature difference required to obtain enough voltage to activate the tag is $8{ }^{\circ} \mathrm{C}$. The result of the oxygen measurement is presented to the user by means of two LEDs that inform if the concentration lies below or above a predefined security limit. Thanks to this approach, the interaction with a nontrained user becomes easier and friendlier, since the information is transmitted in a visual way where the activation of a simple LED allows the user to know if the conditions inside the package are optimal.

An ultralow consumption measurement electronics was designed suitable to be supplied from the low power generated by the Peltier module. For this purpose, the electronics proposed was purely analog, and it was based on the use of a new color sensor aimed to capture only red emission. This color sensor generated an output current in the range of $\mu \mathrm{A}$ in response to the intensity of the incident light. The complete circuit required only $743 \mu \mathrm{W}$ to operate.

The proposed system presented a resolution of $0.02 \%$ in the range of interest $0 \%-5 \%$ of $\mathrm{O}_{2}$ and an LOD of $0.007 \%$, which can make the instrument suitable to be used in IP and AP technologies.

\section{REFERENCES}

[1] J. H. Han, Innovations in Food Packaging. New York, NY, USA: Academic, 2013.

[2] Commission Regulation (EC) No 450/2009, of 29 May 2009 on Active and Intelligent Materials and Articles Intended to Come into Contact With Food, Eur. Food Saf. Authority, Parma, Italy, 2009, pp. 3-11.

[3] B. A. Blakistone, Principles and Applications of Modified Atmosphere Packaging of Foods, 2nd ed. Gaithersburg, MD, USA: Aspen, 1999.

[4] S. Yildirim et al., "Active packaging applications for food," Comprehensive Rev. Food Sci. Food Saf., vol. 17, no. 1, pp. 165-199, Jan. 2018.

[5] J. K. S. Møller, J. S. Jensen, M. B. Olsen, L. H. Skibsted, and G. Bertelsen, "Effect of residual oxygen on colour stability during chill storage of sliced, pasteurised ham packaged in modified atmosphere," Meat Sci., vol. 54, no. 4, pp. 399-405, Apr. 2000.

[6] I. Van Bree et al., "Modelling the degradation kinetics of vitamin C in fruit juice in relation to the initial headspace oxygen concentration," Food Chem., vol. 134, no. 1, pp. 207-214, Sep. 2012. 
[7] J. C. Rickman, D. M. Barrett, and C. M. Bruhn, "Nutritional comparison of fresh, frozen and canned fruits and vegetables. Part 1. Vitamins C and B and phenolic compounds," J. Sci. Food Agric., vol. 87, no. 6, pp. 930-944, Apr. 2007.

[8] F. Rodrigues, M. Côrte-Real, C. Leão, J. P. van Dijken, and J. T. Pronk, "Oxygen requirements of the food spoilage yeast Zygosaccharomyces bailii in synthetic and complex media," Appl. Environ. Microbiol., vol. 67, no. 5, pp. 2123-2128, May 2001.

[9] D. Gibis and K. Rieblinger, "Oxygen scavenging films for food application," Procedia Food Sci., vol. 1, pp. 229-234, 2011.

[10] R. S. Cruz, G. P. Camilloto, and A. C. dos Santos Pires, "Oxygen scavengers: An approach on food preservation," in Structure and Function of Food Engineering. IntechOpen, 2012.

[11] A. D. Bouletis, I. S. Arvanitoyannis, and D. M. Ntionias, "Application of modified atmosphere packaging on quality of selected vegetables: A review," in Application of Modified Atmosphere Packaging on Quality of Selected Vegetables. Cham, Switzerland: Springer, 2012, pp. 1-88.

[12] L. Jacxsens, F. Devlieghere, C. Van der Steen, and J. Debevere, "Effect of high oxygen modified atmosphere packaging on microbial growth and sensorial qualities of fresh-cut produce," Int. J. Food Microbiol., vol. 71, nos. 2-3, pp. 197-210, Dec. 2001.

[13] A. Mills, "Oxygen indicators and intelligent inks for packaging food," Chem. Soc. Rev., vol. 34, no. 12, pp. 1003-1011, 2005.

[14] A. W. Hempel, M. G. O’Sullivan, D. B. Papkovsky, and J. P. Kerry, "Use of smart packaging technologies for monitoring and extending the shelflife quality of modified atmosphere packaged (MAP) bread: Application of intelligent oxygen sensors and active ethanol emitters," Eur. Food Res. Technol., vol. 237, no. 2, pp. 117-124, Aug. 2013.

[15] A. Mills, K. Lawrie, J. Bardin, A. Apedaile, G. A. Skinner, and C. O'Rourke, "An $\mathrm{O}_{2}$ smart plastic film for packaging," Analyst, vol. 137, no. 1, pp. 106-112, 2012.

[16] C. H. T. Vu and K. Won, "Novel water-resistant UV-activated oxygen indicator for intelligent food packaging," Food Chem., vol. 140, nos. 1-2, pp. 52-56, 2013.

[17] K. Won, N. Y. Jang, and J. Jeon, "A natural component-based oxygen indicator with in-pack activation for intelligent food packaging," J. Agric. Food Chem., vol. 64, no. 51, pp. 9675-9679, 2016.

[18] J. Ehgartner, H. Wiltsche, S. M. Borisov, and T. Mayr, "Low cost referenced luminescent imaging of oxygen and $\mathrm{pH}$ with a 2-CCD colour near infrared camera," Analyst, vol. 139, no. 19, pp. 4924-4933, 2014

[19] F. C. O’Mahony, T. C. O’Riordan, N. Papkovskaia, V. I. Ogurtsov, J. P. Kerry, and D. B. Papkovsky, "Assessment of oxygen levels in convenience-style muscle-based sous vide products through optical means and impact on shelf-life stability," Packag. Technol. Sci. Int. J., vol. 17, no. 4, pp. 225-234, 2004.

[20] M. Fitzgerald et al., "Nondestructive monitoring of oxygen profiles in packaged foods using phase-fluorimetric oxygen sensor," J. Food Sci., vol. 66, no. 1, pp. 105-110, 2001.

[21] A. Mills, C. Tommons, R. T. Bailey, M. C. Tedford, and P. J. Crilly, "UV-Activated Luminescence/Colourimetric $\mathrm{O}_{2}$ indicator," Int. J. Photoenergy, vol. 2008, Nov. 2007, Art. no. 547301.

[22] J. C. C. Rodriguez, M. A. P. Garcia, M. G. Vega, and F. J. Ferrero, "Measurement of low oxygen concentrations by phosphorescence lifetime using optical fibers," IEEE Trans. Instrum. Meas., vol. 48, no. 5, pp. 949-955, Oct. 1999.

[23] S. Banerjee, C. Kelly, J. P. Kerry, and D. B. Papkovsky, "High throughput non-destructive assessment of quality and safety of packaged food products using phosphorescent oxygen sensors," Trends Food Sci. Technol., vol. 50, pp. 85-102, Apr. 2016.

[24] G. Fuertes, I. Soto, R. Carrasco, M. Vargas, J. Sabattin, and C. Lagos, "Intelligent packaging systems: Sensors and nanosensors to monitor food quality and safety," J. Sensors, vol. 2016, Sep. 2016, Art. no. 4046061.

[25] F. Bibi, C. Guillaume, N. Gontard, and B. Sorli, "A review: RFID technology having sensing aptitudes for food industry and their contribution to tracking and monitoring of food products," Trends Food Sci. Technol., vol. 62, pp. 91-103, Apr. 2017.

[26] P. Escobedo et al., "Flexible passive near field communication tag for multigas sensing," Anal. Chem., vol. 89, no. 3, pp. 1697-1703, 2017.

[27] A. Siddiqui, R. Mahboob, and T. Islam, "A passive wireless tag with digital readout unit for wide range humidity measurement," IEEE Trans. Instrum. Meas., vol. 66, no. 5, pp. 1013-1020, May 2017.

[28] K. B. Biji, C. N. Ravishankar, C. O. Mohan, and T. K. S. Gopal, "Smart packaging systems for food applications: A review," J. Food Sci. Technol., vol. 52, no. 10, pp. 6125-6135, Oct. 2015.
[29] R. Zhu, M. Desroches, B. Yoon, and T. M. Swager, "Wireless oxygen sensors enabled by Fe(II)-polymer wrapped carbon nanotubes," ACS Sensors, vol. 2, no. 7, pp. 1044-1050, Jul. 2017.

[30] P. Escobedo, I. M. P. de Vargas-Sansalvador, M. Carvajal, L. F. CapitánVallvey, A. J. Palma, and A. Martínez-Olmos, "Flexible passive tag based on light energy harvesting for gas threshold determination in sealed environments," Sens. Actuators B, Chem., vol. 236, pp. 226-232, Nov. 2016.

[31] I. Okura, Photosensitization of Porphyrins and Phthalocyanines. New York, NY, USA: Gordon and Breack Science, 2000.

G. Korotcenkov, Handbook of Gas Sensor Materials: Properties, Advantages and Shortcomings for Applications, vol. 2. Springer, 2014.

[33] Y. Amao, T. Miyashita, and I. Okura, "Optical oxygen sensing based on the luminescence change of metalloporphyrins immobilized in styrene-pentafluorostyrene copolymer film," Analyst, vol. 125, no. 5, pp. 871-875, 2000.

[34] A. Martínez-Olmos, J. Fernández-Salmerón, N. Lopez-Ruiz, A. R. Torres, L. F. Capitan-Vallvey, and A. J. Palma, "Screen printed flexible radiofrequency identification tag for oxygen monitoring," Anal. Chem., vol. 85, no. 22, pp. 11098-11105, 2013.

[35] Linear Technology Corporation. LTC3108 Datasheet. Accessed: May 2, 2018. [Online]. Available: http://www.analog.com/media/ en/technical-documentation/data-sheets/3108fc.pdf

[36] S.-H. Huang, Y.-H. Hsu, C.-W. Wu, and C.-J. Wu, "Light-addressable measurements of cellular oxygen consumption rates in microwell arrays based on phase-based phosphorescence lifetime detection," Biomicrofluidics, vol. 6, no. 4, 2012, Art. no. 044118.

[37] N. López-Ruiz, D. Hernández-Bélanger, M. A. Carvajal, L. F. Capitán-Vallvey, A. J. Palma, and A. Martínez-Olmos, "Fast lifetime and amplitude determination in luminescence exponential decays," Sens. Actuator B, Chem., vol. 216, pp. 595-602, 2015.

[38] D. A. Armbruster and T. Pry, "Limit of blank, limit of detection and limit of quantitation," Clin. Biochem. Rev., vol. 29, no. 1, pp. S49-S52, 2008.

Pablo Escobedo Araque was born in Jaén, Spain, in 1989. He received the M.Sc. degree in telecommunication engineering and electronics engineering from the University of Granada, Granada, Spain, in 2012 and 2013, respectively, and the master's degree in computer and network engineering in 2014. He is currently pursuing the Ph.D. degree with the ECSens Group, Department of Electronics and Computer Technology, University of Granada, under the National Scholarship on the design and development of printed RFID labels with sensing capabilities.

Isabel M. Pérez de Vargas-Sansalvador received the M.Sc. degree and the $\mathrm{Ph} . \mathrm{D}$. degree in chemistry from the University of Granada, Granada, Spain, in 2007 and 2011, respectively.

She is currently a Post-Doctoral Researcher with the University of Granada. Her current research interests include optical gas sensing and microfluidics.

Nuria López-Ruiz was born in Barcelona, Spain, in 1985. She received the B.S. degree in telecommunications engineering, the B.S. degree in electronic engineering, the M.Sc. degree in telecommunications engineering, and the Ph.D. degree in information and communication technologies from the University of Granada, Granada, Spain, in 2008, 2009, 2010, and 2014, respectively.

She is currently an Assistant Professor with the Carlos III University of Madrid, Madrid, Spain. Her current research interests include the study of different colorimetric and optical sensors for environmental measurements, and also the development of portable electronic instrumentation and smartphone applications associated with them. 
Luis Fermín Capitán-Vallvey received the B.Sc. and Ph.D. degrees in chemistry from the Faculty of Sciences, University of Granada, Granada, Spain, in 1973 and 1986, respectively.

In 1983, he founded the Solid Phase Spectrometry Group (GSB) and, in 2000, together with Prof. P. López, the interdisciplinary group, ECsens, University of Granada, which includes chemists, physicists, and electrical and computer engineers, at the University of Granada. He is currently a Full Professor of analytical chemistry with the University of Granada. He has authored nearly 350 peer-reviewed scientific papers, six books, and 25 book chapters, and holds six patents. His current research interests include design, development, fabrication of sensors and portable instrumentation for environmental, health, and food analysis and monitoring, printing chemical sensor, and capillary-based microfluidic devices.

Alberto J. Palma received the B.S. and M.Sc. degrees in physics and the Ph.D. degree from the University of Granada, Granada, Spain, in 1991 and 1995, respectively.

$\mathrm{He}$ is currently a Full Professor with the Department of Electronics and Computer Technology, University of Granada. Since 1992, he has been working on trapping of carriers in different electronic devices (diodes and MOS transistors), including characterization and simulation of capture cross sections, random telegraph noise, and generation-recombination noise in devices. His current research interests include design, development, fabrication of sensors and portable electronic instrumentation for environmental, biomedical, and food analysis and monitoring, and printing sensors on flexible substrates with processing electronics using inkjet and screen-printing technologies.
Miguel. A. Carvajal Rodríguez was born in Granada, Spain, in 1977 $\mathrm{He}$ received the M.Sc. degree in physics, the M.Sc. degree in electronic engineering, and the Ph.D. degree in electronic engineering, focusing on the development of a dosimeter system based on commercial MOSFETs, from the University of Granada, Granada, in 2000, 2002, and 2007, respectively.

$\mathrm{He}$ is currently a Tenured Professor with the University of Granada. His current research interests include the effects of irradiation and post-irradiation in MOSFET transistors, gas sensor, and electrochemiluminescent sensors and their applications to handheld instrumentation.

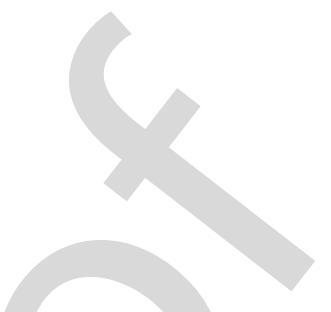

Antonio Martínez-Olmos was born in Granada, Spain, in 1980. He received the M.Sc. degree and the $\mathrm{Ph} . \mathrm{D}$. degree in electronic engineering from the University of Granada, Granada, in 2003 and 2009, respectively.

$\mathrm{He}$ is currently a Tenured Professor with the University of Granada. His current research interests include the development of optical sensors for environmental and biological measurements. 